\title{
Sensors vs. experts - A performance comparison of sensor-based fall risk assessment vs. conventional assessment in a sample of geriatric patients
}

\author{
Michael Marschollek ${ }^{1 *}$, Anja Rehwald ${ }^{1}$, Klaus-Hendrik Wolf², Matthias Gietzelt², Gerhard Nemitz ${ }^{3}$, \\ Hubertus Meyer zu Schwabedissen ${ }^{3}$ and Mareike Schulze ${ }^{1}$
}

\begin{abstract}
Background: Fall events contribute significantly to mortality, morbidity and costs in our ageing population. In order to identify persons at risk and to target preventive measures, many scores and assessment tools have been developed. These often require expertise and are costly to implement. Recent research investigates the use of wearable inertial sensors to provide objective data on motion features which can be used to assess individual fall risk automatically. So far it is unknown how well this new method performs in comparison with conventional fall risk assessment tools. The aim of our research is to compare the predictive performance of our new sensor-based method with conventional and established methods, based on prospective data.
\end{abstract}

Methods: In a first study phase, 119 inpatients of a geriatric clinic took part in motion measurements using a wireless triaxial accelerometer during a Timed Up\&Go (TUG) test and a $20 \mathrm{~m}$ walk. Furthermore, the St. Thomas Risk Assessment Tool in Falling Elderly Inpatients (STRATIFY) was performed, and the multidisciplinary geriatric care team estimated the patients' fall risk. In a second follow-up phase of the study, 46 of the participants were interviewed after one year, including a fall and activity assessment. The predictive performances of the TUG, the STRATIFY and team scores are compared. Furthermore, two automatically induced logistic regression models based on conventional clinical and assessment data (CONV) as well as sensor data (SENSOR) are matched.

Results: Among the risk assessment scores, the geriatric team score (sensitivity 56\%, specificity $80 \%$ ) outperforms STRATIFY and TUG. The induced logistic regression models CONV and SENSOR achieve similar performance values (sensitivity $68 \% / 58 \%$, specificity $74 \% / 78 \%$, AUC $0.74 / 0.72,+$ LR $2.64 / 2.61$ ). Both models are able to identify more persons at risk than the simple scores.

Conclusions: Sensor-based objective measurements of motion parameters in geriatric patients can be used to assess individual fall risk, and our prediction model's performance matches that of a model based on conventional clinical and assessment data. Sensor-based measurements using a small wearable device may contribute significant information to conventional methods and are feasible in an unsupervised setting. More prospective research is needed to assess the cost-benefit relation of our approach.

\section{Background}

It is well-known that fall events constitute an important factor with regard to mortality, morbidity and costs in our aging population. These events have a high incidence especially in the elderly: $25.1 \%$ of the men and $37 \%$ of the

\footnotetext{
* Correspondence: Michael.Marschollek@plri.de

'Hanover Medical School, Peter L. Reichertz Institute for Medical Informatics,

Carl-Neuberg-Str. 1, Hanover, 30625, Germany

Full list of author information is available at the end of the article
}

women aged 65 years and above fall at least once within 12 months [1]. The highest incidence is reported for geriatric inpatients [1], which often have several risk factors [2] at the same time and suffer from multiple diseases: The prevalence rate of five or more somatic diseases for persons aged 70 years and above has been reported in the Berlin Aging Study to be 88\% [3]. As fall events and their consequences are very costly - an estimated annual $19.2 \$$ billion in the U.S. [4] - preventive measures have 
been investigated intensively [5]. These measures themselves are costly, so that two predominant questions are: Who should be treated in the first place, and who should receive which kind of preventive measure?

In order to identify persons at risk to fall down - thus being eligible for preventive treatment - many risk assessment tools, e.g. the Timed UpEGo test (TUG) [6] or the St. Thomas Risk Assessment Tool in Falling Elderly Inpatients (STRATIFY) [7] have been developed and evaluated in a multitude of studies. Comprehensive reviews can be found e.g. in $[2,8,9]$. Several tests have also been used to predict falls in outpatients, often with a specific group of patients. Kikuchi et al. report that, in a prospective study with 79 patients having a diagnosis of cognitive impairment and lasting 12 months, only their fall-predicting score, a self-answered 21-item questionnaire, was predictive of future falls [10], but not e.g. the TUG. The latter test was, in contrast, found as the only predictive parameter for falls in patients after hip surgery in a 6-month prospective study by Kristensen et al. [11] Hale et al. found that mobility scores were not associated with falls in a 12-month prospective study with 120 geriatric outpatients, but history of falls was [12]. Oliver et al. conclude that even the best tools are not able to identify the majority of fallers [9]. Keeping this in mind along with the often time-consuming nature of fall risk assessment tests (e.g. the Performance-Oriented Mobility Assessment, POMA [13]) that frequently require expert knowledge, several research groups have developed the idea to perform a sensorbased automatic or semi-automatic assessment using wearable inertial sensors [14-17]. Apart from offering continuous and objective data, this approach may also serve to detect fall events once they have happened, being aware of the fact that many falls go by undetected and a person may lie injured hours or even days in her or his flat. Despite promising first results of this sensorbased approach developed by the authors [18], it remains unclear how well the new methods perform in comparison with conventional fall risk assessment tools.

Therefore, the aim of our research work for this paper is to examine the predictive performance of our new sensor-based method for fall risk assessment in comparison with conventional and established methods. The comparison is based on one-year follow-up data obtained in a prospective study.

\section{Methods}

\section{General approach}

We recruited a sample of geriatric patients - who are known to have the highest risk of falling [1] - and performed selected conventional assessment tests which are used to determine fall risk. Furthermore, fall risk was assessed by the interdisciplinary geriatric care team (physicians, nurses, physiotherapists, occupational therapists) of the Department for Geriatric Medicine at Braunschweig Medical Center in Germany. In addition to these measures, a sensor-based assessment of fall risk was performed, which employs gait and motion parameters obtained during a Timed Up\&Go test and a $20 \mathrm{~m}$ walk during the patients' hospital stay. These parameters are: kinetic energy, pelvic sway along the transversal axis, standard deviation of gait periodicity, mean step duration, step length, number of steps during Timed Up \& Go test and a number of spectral density distribution parameters such as the frequency of the most prominent spectral density peak. The sensor equipment used, the methods for parameter extraction from raw data and the generation of prediction models are explained in detail in $[18,19]$. The study protocol has been approved by the Hanover Medical School ethics committee.

\section{Study population}

The study population consisted of geriatric inpatients of the Braunschweig Medical Center's Department for Geriatric Medicine who met the following inclusion criteria:

- admission between April $24^{\text {th }}$ and October $18^{\text {th }}$, 2007

- ability to stand up and walk

- written consent (also by a third party) to take part in the follow-up interviews

Although 119 patients took part in the first phase of the study, including the geriatric fall risk assessment tests and the sensor measurements, only 50 were able or willing to participate in the follow-up investigation (37 women and 13 men). The reasons for drop-outs were: death $(n=17)$, untraceable ( $n=13$ ), no answer to the letter asking for written consent $(n=27)$, rejecting the telephone interview despite previous consent ( $n=4)$, and several other problems such as deafness or cognitive impairment $(n=8)$ [18]. Four of the motion sensor data sets were corrupted, so that altogether 46 persons (mean age 81.3 years) could be included in the fall risk assessment performance comparison study. With regard to the high number of dropouts we compared the group of participants with the drop-outs in respect of relevant clinical parameters and fall risk assessments (age, body mass index, Timed Up\&Go test, geriatric team risk score as described below, STRATIFY score and Barthel index score). There were no statistically significant differences between the two groups, except for a higher STRATIFY score in the drop-outgroup ( 2.8 vs. $2.3, \mathrm{p}=0.036)$. As mental alteration is one of the STRATIFY items, this result may indicate that persons with cognitive impairments were more likely to decline to give written consent or even to die. In a further 
analysis of STRATIFY sub-items, however, no significant group differences could be identified.

\section{Conventional geriatric fall risk assessment tests and additional clinical parameters}

Several clinical parameters as well as geriatric assessment tests were performed by clinical staff members on admission to the geriatric department. The clinical parameters were age, sex and body mass index (BMI). Furthermore, the St. Thomas Risk Assessment Tool in Falling Elderly Inpatients (STRATIFY) score [7] and the Barthel index [20] were assessed, and the Timed UpEGo test [6] was performed plus an additional $20 \mathrm{~m}$ walkway.

Apart from these standardized methods, the multidisciplinary geriatric care team assessed individual fall risk using a self-developed rating scale, which simply consists of three values: no risk, low risk and high risk.

In addition to the above-mentioned assessments which are performed during hospital stays, in the second phase of the study - conducted one year after the first phase - we used telephone interviews in order to assess fall events within the last year as well as general physical activity levels. A detailed account of physical activity levels among fallers and non-fallers can be found in [21]. The fallrelated interviews were structured in accordance with the Prevention of Falls Network Europe (ProFaNE) consensus criteria [22] and are described in more detail in [18].

\section{Sensor equipment}

During the inpatient phase of the study, all participants wore a wireless triaxial accelerometer system (Freescale RD3152MMA7260Q) on a belt around the waist (Figure 1) during the Timed Up\&Go test and the $20 \mathrm{~m}$ walkway in the physiotherapy department of the clinic. The data were transmitted to a PC and stored for processing.

\section{Classification model induction and evaluation}

In order to evaluate the predictive performance of the several fall risk assessment tools, we chose a two-fold approach. First of all, we used the two dedicated fall risk assessment scales - the STRATIFY score and the Timed Up\&Go test - along with the care team score to distinguish between fallers and non-fallers within the year of follow-up. The cut-off points were set as proposed by the developers in their original publications: $\geq 2$ points for the STRATIFY score [7], > 20s for the Timed Up\&Go test [6] and no risk vs. low/high risk for the team score. For each score, we constructed a contingency table and calculated sensitivity (SENS), specificity (SPEC), positive (PPV) and negative predictive values (NPV) as well as overall classification accuracy (ACC).

In a second step, we used all clinical and fall risk assessment parameters to automatically induce a classification model (model CONV). The same has been done

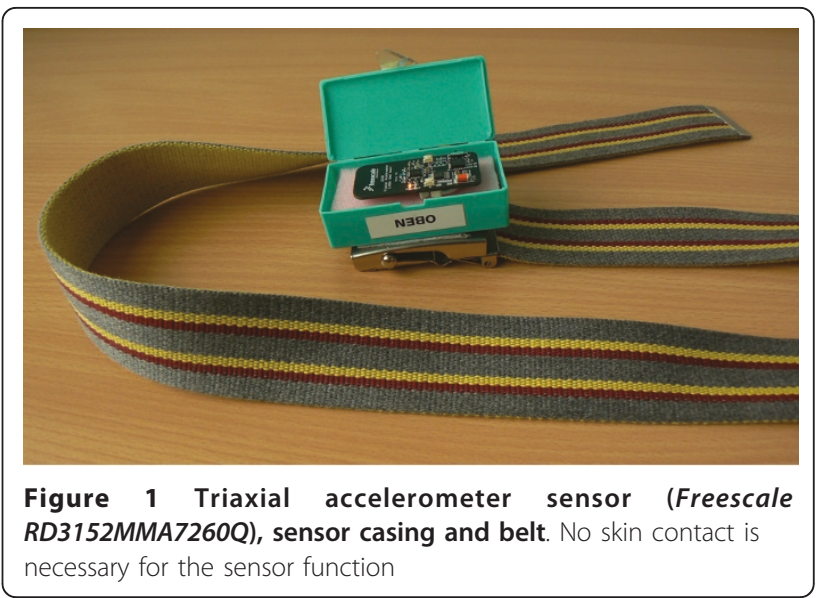

previously for the parameters that were extracted from sensor data and overall activity (model SENSOR) [18]. We chose to use a logistic regression model based on its known properties of stability and performance in small data sets and employed the Open Source toolkit Waikato Environment for Knowledge Analysis (WEKA, version 3.7.1, simple logistic algorithm; parameters: error on probabilities $=$ true, heuristic stop $=50$, maximum number of iterations for LogitBoost $=5000$, use cross validation = true, no weight trimming) [23]. For model induction, the binary attribute fall within the last year (yes/no) was used. The multi-parameter data sets were pre-processed using a feature selection algorithm in order to exclude parameters with low information (WEKA, version 3.7.1, wrapper subset evaluator, employing the simple logistic regression algorithm as described above). All automatically induced classification models were evaluated using a ten times ten-fold cross-validation procedure, and SENS, SPEC, PPV, NPV, ACC and the area under the curve (AUC) were calculated. Furthermore, we computed the each model's Brier score as a performance measure [24], which is the mean squared difference between the observed outcome and the predicted probability for each instance in the data set. Finally, the positive likelihood ratios $(+\mathrm{LR})$ of all models were calculated as an additional performance measure that spans the whole of each contingency table.

\section{Results}

Tables 1, 2 and 3 show the results of the single fall risk assessment tests for the prediction of actual fall events within a year after discharge from the geriatric ward. In Table 4, all +LR values are presented. The STRATIFY score (Table 1), a dedicated fall risk tool, has an overall classification accuracy of $48 \%$ with a good sensitivity of $79 \%$ but a low specificity of $26 \%$. While the NPV is $63 \%$, the PPV is only $43 \%$, meaning that a positive assessment result does not predict actual falls well. 
Table 1 Classification results and contingency table for the STRATIFY score [7] (cut-off point $\geq 2$ points)

\begin{tabular}{cccccc}
\hline \multicolumn{5}{c}{ STRATIFY score } \\
\hline \multicolumn{5}{c}{ contingency table } \\
\hline classification accuracy & $48 \%$ & & fall within one year \\
\hline sensitivity & $79 \%$ & & yes & no & Sum \\
\hline specificity & $26 \%$ & pred. yes & 15 & 20 & 35 \\
\hline negative predictive value & $63 \%$ & pred. no & 4 & 7 & 11 \\
\hline positive predictive value & $43 \%$ & sum & 19 & 27 & 46 \\
\hline
\end{tabular}

The Timed UpEGo test results in Table 2 show an overall classification accuracy of $50 \%$, where a high sensitivity of $90 \%$ is pitted against a very low specificity of $22 \%$. Similar to the STRATIFY score results, the NPV is slightly higher (75\%) than the PPV (45\%).

The geriatric team's fall risk assessment score (Table 3) shows more balanced, though not really much better results: A classification accuracy of $55 \%$ is accompanied by a sensitivity of $63 \%$ and a specificity of $50 \%$. The NPV is $68 \%$ and the PPV is $44 \%$. The $+\mathrm{LR}$ values (Table 4) of all three simple fall risk assessments $(1.07,1.15$ and 1.25) confirm their low predictive power, yet among these the team score has the highest hit ratio.

The automatically generated classification model CONV (Table 5) based on clinical and geriatric assessment data shows markedly better performance values than the three previous tests: The classification accuracy is $72 \%$ with a sensitivity of $68 \%$ and a specificity of $74 \%$. Furthermore, both the NPV (77\%) and the PPV (65\%) are balanced and on a fair level. The overall good performance of this model is also shown by both the Brier score of 0.2 , an AUC of 0.74 and a statistically significant + LR value of 2.64 (Table 4).

The classification model SENSOR (Table 6, [18]) matches the CONV model in its measures: Classification accuracy is $70 \%$, with a sensitivity of $58 \%$ and a specificity of $78 \%$. NPV (72\%) and PPV (65\%) are also level. The $+\mathrm{LR}$ value of 2.61 , however, does not reach statistical significance due to the broader confidence interval.

Table 2 Classification results and contingency table for the Timed Up\&Go test [6] (cut-off point $>20$ s)

\begin{tabular}{|c|c|c|c|c|c|}
\hline \multicolumn{6}{|c|}{ Timed Up\&Go test } \\
\hline & & & \multicolumn{3}{|c|}{ contingency table } \\
\hline classification accuracy & $50 \%$ & & \multicolumn{3}{|c|}{ fall within one year } \\
\hline sensitivity & $90 \%$ & & yes & no & $\overline{\text { Sum }}$ \\
\hline specificity & $22 \%$ & pred. yes & 17 & 21 & 38 \\
\hline negative predictive value & $75 \%$ & pred. no & 2 & 6 & 8 \\
\hline positive predictive value & $45 \%$ & sum & 19 & 27 & 46 \\
\hline
\end{tabular}

Table 3 Classification results and contingency table for multidisciplinary geriatric team fall risk score (4 missing values)

\begin{tabular}{cccccc}
\hline \multicolumn{5}{l}{ TEAM assessment } \\
\hline \multicolumn{5}{c}{5} & \multicolumn{4}{l}{ contingency table } \\
\hline classification accuracy & $55 \%$ & & fall within one year \\
\hline sensitivity & $63 \%$ & & yes & no & Sum \\
\hline specificity & $50 \%$ & pred. yes & 10 & 13 & 23 \\
\hline negative predictive value & $68 \%$ & pred. no & 6 & 13 & 19 \\
\hline positive predictive value & $44 \%$ & sum & 16 & 26 & 42 \\
\hline
\end{tabular}

\section{Discussion}

The performances of the simple fall risk assessment tools used in this study - the STRATIFY score, the Timed Up\&Go (TUG) test and the geriatric care team rating - are limited. In a recent meta-analysis Oliver et al., who have developed the STRATIFY score, report the following values for geriatric patients: SENS 67.2\%, SPEC 51.2\%, PPV 23.1\% and NPV 86.5\% ( $\mathrm{n}=1285$ patients, four different studies) [9]. Kim et al. have also evaluated the STRATIFY score, albeit with a much younger cohort (mean age 56 years, $\mathrm{n}=5489$ patients, 60 fallers), and find: SENS 55\%, SPEC 75.3\%, PPV $2.4 \%$ and NPV 99.3\% [25]. Our results show a slightly worse performance than was reported in the meta-analysis by Oliver et al. [9]. This may well be due to our very small sample size. The same applies to the Timed Up\&Go test. Nordin et al. have studied the predictive validity of the TUG in 183 patients with a mean age of 84.3 years and a cut-off point of 20s [26]. They report a sensitivity of $79 \%$ and a specificity of $32 \%$. In the large Tromsø study Thrane et al. find sensitivity values of $44-14 \%$ and specificity values of $58-90 \%$ for the TUG, depending on the choice of the cut-off points (here between 12 and 17s) [27]. Kristensen et al. report SENS 95\%, SPEC 35\%, PPV 41\%, NPV 93\%, +LR 1.5, -LR 0.1 for the TUG's predictive performance for patients after hip surgery (mean age 81 years, $\mathrm{n}=59$ patients, 19 fallers, cut-off value 24s) [11]. Our results (Table 2) also show a remarkable sensitivity of $90 \%$ for the TUG, yet the specificity is way too low for a screening test. This is confirmed by the low +LR value of 1.15 .

Table 4 +LR values of all five classification models including the confidence intervals

\begin{tabular}{lcc}
\hline model name & +LR value & 95\% confidence interval \\
\hline STRATIFY score & 1.07 & $0.71-1.61$ \\
\hline Timed Up\&Go test & 1.15 & $0.83-1.59$ \\
\hline Team Assessment & 1.25 & $0.63-2.49$ \\
\hline model CONV & 2.64 & $1.07-6.5$ \\
\hline model SENSOR & 2.61 & $0.94-7.26$ \\
\hline
\end{tabular}


Table 5 Classification results and contingency table for logistic regression model based on clinical data and fall risk assessment tests

\begin{tabular}{cccccc}
\hline \multicolumn{5}{c}{ model CONV } \\
\hline classification accuracy & $72 \%$ & & & \\
\hline sensitivity & $68 \%$ & & \multicolumn{4}{c}{ contingency table } \\
\hline specificity & $74 \%$ & & fall within one year \\
\hline negative predictive value & $77 \%$ & & yes & no & Sum \\
\hline positive predictive value & $65 \%$ & pred. yes & 13 & 7 & 20 \\
\hline Brier score & 0.20 & pred. no & 6 & 20 & 26 \\
\hline AUC & 0.74 & sum & 19 & 27 & 46 \\
\hline
\end{tabular}

Both tests are very simple to perform, either by history taking or by conducting a simple physical test, and both take only a couple of minutes. Therefore, these tests may serve well - and in fact are frequently used - as general screening methods, if necessary followed by more complex, multimodal assessment inventories such as the Physiological Profile Assessment (PPA) [28].

The geriatric care team fall risk score may be perceived as a very subjective measure, yet it represents the professional opinion of several experienced experts that is very likely based on an intuitive understanding of the complex concept 'fall risk' as well as on a multitude of observations of a certain patient. This solid foundation is reflected by the fair performance values of this score, which are the most balanced of the three simple tests. Similar results have been found in [26], where 'global rating of fall risk' (low/high) by staff members achieved a sensitivity of $56 \%$ and a specificity of $80 \%$.

The automatically induced model CONV (Table 5) shows better performance values than all of the above tests. This is of course due to the approach of including basic clinical data such as sex, BMI and age in the induction process, but also to the combination of different assessment methods ranging from a physical test (TUG) over a measure of daily activity capability (Barthel index) to a fall risk score (STRATIFY). In the induction process, the most relevant parameters or scores are identified and

Table 6 Classification results and contingency table for logistic regression model based on sensor data and longterm physical activity level

\begin{tabular}{cllccc}
\hline \multicolumn{5}{l}{ model SENSOR } \\
\hline classification accuracy & $70 \%$ & & & \\
\hline sensitivity & $58 \%$ & & \multicolumn{3}{c}{ contingency table } \\
\hline specificity & $78 \%$ & & fall within one year \\
\hline negative predictive value & $72 \%$ & & yes & no & Sum \\
\hline positive predictive value & $65 \%$ & pred. yes & 11 & 6 & 17 \\
\hline Brier score & 0.21 & pred. no & 8 & 21 & 29 \\
\hline AUC & 0.72 & sum & 19 & 27 & 46 \\
\hline
\end{tabular}

included, so that performance is optimized. The multitude of candidate parameters may capture the multifactorial concept of fall risk more adequately than a single test. The performance measures show that CONV can identify most of the fallers and non-fallers correctly, based on their one-year outcome. Thus, this model could be suitable as a screening test for geriatric patients, facilitating the prescription of preventive measures.

When compared to the previously computed SENSOR model, which is based merely on accelerometer sensor data and overall activity levels, we can state that this model performs almost equally well than the CONV model. The Brier scores ( 0.21 vs. 0.2$)$ are nearly the same, as are the AUC (0.74 vs. 0.72$)$ and $+\mathrm{LR}$ values (2.64 vs. 2.61). Therefore, regarding our preliminary results we may conclude that by using sensor data which may be recorded over extended periods of time during normal daily activities with a small and unobtrusive device - we can match the performance of conventional methods with regard to fall risk assessment in a sample of geriatric patients. The advantage of our approach is of course the absent necessity of an expert physiotherapist, nurse or physician to perform the assessment. This could be done by the wearable device itself, using long-term motion data along with the developed algorithm. Potential drawbacks of our approach are possible technical failures such as data loss from the accelerometer device, acceptance issues, limited battery lifetime and the current lack of technical infrastructures (e.g. sensor-enhanced health information systems $[29,30])$. Technical equipment and infrastructures are of course costly, but so are falls and their consequences in the first place.

Future prospective studies will have to be conducted with more patients and over an even longer period of time, evaluating the validity of our approach and our preliminary results in an independent patient sample on the one hand and the cost-benefit relation on the other hand. From an ethics point of view, however, one might argue that every fall that is avoided is a big benefit for the individual.

From a technical point of view, more research work is needed to look into the potential predictive parameters that can be extracted from sensor data [31]. Furthermore, in this study we have not considered any information from the patients' electronic health records (EHRs) [32], such as diagnoses or additional history. Considering the multi-factorial aetiology of falls [33], our sensorbased information may well be used in combination with resp. as supplement to conventional geriatric assessment tools and other clinical data.

\section{Limitations}

Our sample size is small and this - in comparison with large trials evaluating the conventional methods-limits 
the generalizability of our results. So does the fact that, due to the sample size, we cannot use separate training and test data sets for model induction. Nevertheless, we have chosen a well-established procedure to avoid overfitting of our models, namely ten times, ten-fold crossvalidation. The necessity for written consent to be returned by the patients via surface mail may have led to the exclusion of persons with cognitive impairments, even though consent by a third party was an option. Furthermore, in our follow-up study telephone interviews were used to identify fall events. This approach is errorprone, as many factors (e.g. cognitive impairment) may affect the recall of such events. In addition to this, considering the group of patients, within a period of twelve months risk factors may have changed significantly. Therefore, daily recordings as well as more frequent interviews, e.g. on a monthly basis as recommended in ProFaNE consensus criteria recommendation no.7 [22], might have reduced the error rate, but have not been performed due to a lack of resources. Hence, in our future prospective studies, we will include a more stringent monitoring approach.

From an economic perspective, it remains unclear if the prediction results are good enough to justify the implementation of costly preventive measures for the false positives [5]. A cost-benefit analysis should be conducted, comparing direct and indirect costs of fall events with those of preventive measures. Furthermore, despite promising preliminary studies (e.g. [34-36]), the patients' acceptance of long-term monitoring should be assessed, e.g. using the Sensor Acceptance Model [37].

\section{Conclusions}

To the authors' knowledge, this is the first study to compare sensor-based fall risk assessment to conventional assessment tools in a prospective long-term setting. Our preliminary results indicate that a fall risk model based on accelerometer sensor data performs almost as well as a model that is derived from conventional geriatric assessment data. Therefore we may conclude that such a model can provide relevant information and thus - considering the multi-factorial aetiology of fall events - could be valuable not as a replacement of professional assessment scores and tools, but as a supplementary method which is feasible to be used outside of a supervised clinical environment.

\footnotetext{
Acknowledgements

This publication is supported by the project "Open Access Publizieren" by the Deutsche Forschungsgemeinschaft (DFG). We thank the physiotherapy team of the Department for Geriatric Medicine at the Braunschweig Medical Center for their invaluable support, especially Cornelia Kühling. We thank Wolfram Ludwig for his support in conducting some of the measurements.
}

\section{Author details}

${ }^{1}$ Hanover Medical School, Peter L. Reichertz Institute for Medical Informatics, Carl-Neuberg-Str. 1, Hanover, 30625, Germany. ${ }^{2}$ University of Braunschweig Institute of Technology, Peter L. Reichertz Institute for Medical Informatics, Mühlenpfordtstr. 23, Braunschweig, 38106, Germany. ${ }^{3}$ Braunschweig Medical Center, Department for Geriatric Medicine, Gliesmaroder Straße 29, Braunschweig, 38106, Germany.

\section{Authors' contributions}

MM supervised the study, carried out the data analysis and drafted the manuscript. AR designed and conducted the telephone interviews. KHW participated in drafting the study protocol. MG conducted the sensor data measurements and participated in the data analysis. GN performed the geriatric assessment tests and participated in drafting the study protocol. HMZS conceived of the study and participated in its coordination. MS participated in the data analysis and the computation of performance values. All authors read and approved the final manuscript.

\section{Competing interests}

The authors declare that they have no competing interests.

Received: 7 March 2011 Accepted: 28 June 2011

Published: 28 June 2011

\section{References}

1. von Renteln-Kruse W, Krause T, Dieckmann P, Vogel J: Geriatric patients' mobility status as reflected by the relevant items of the Barthel index and in-hospital falls. J Am Geriatr Soc 2006, 54:1012-1013.

2. Oliver D, Daly F, Martin FC, McMurdo ME: Risk factors and risk assessment tools for falls in hospital in-patients: a systematic review. Age Ageing 2004, 33:122-130.

3. Steinhagen-Thiessen E, Borchelt M: Morbidität, Medikation und Funktionalität im Alter [Morbidity, medication and functional status in the elderly]. In Die Berliner Altersstudie [The Berlin Aging Study]. Edited by: Mayer KU, Baltes PB. Berlin: Akademie Verlag; 1996:151-184.

4. Stevens JA, Corso PS, Finkelstein EA, Miller TR: The costs of fatal and nonfatal falls among older adults. Inj Prev 2006, 12:290-295.

5. Gillespie LD, Robertson MC, Gillespie WJ, Lamb SE, Gates S, Cumming RG, Rowe $\mathrm{BH}$ : Interventions for preventing falls in older people living in the community. Cochrane Database Syst Rev 2009, CD007146.

6. Podsiadlo D, Richardson S: The timed "Up \& Go": a test of basic functional mobility for frail elderly persons. J Am Geriatr Soc 1991 39:142-148.

7. Oliver D, Britton M, Seed P, Martin FC, Hopper AH: Development and evaluation of evidence based risk assessment tool (STRATIFY) to predict which elderly inpatients will fall: case-control and cohort studies. Bmj 1997, 315:1049-1053

8. Gates S, Smith LA, Fisher JD, Lamb SE: Systematic review of accuracy of screening instruments for predicting fall risk among independently living older adults. J Rehabil Res Dev 2008, 45:1105-1116.

9. Oliver D, Papaioannou A, Giangregorio L, Thabane L, Reizgys K, Foster G: A systematic review and meta-analysis of studies using the STRATIFY tool for prediction of falls in hospital patients: how well does it work? Age Ageing 2008, 37:621-627.

10. Kikuchi R, Kozaki K, Iwata A, Hasegawa H, Toba K: Evaluation of risk of falls in patients at a memory impairment outpatient clinic. Geriatr Gerontol Int 2009, 9:298-303.

11. Kristensen MT, Foss NB, Kehlet H: Timed "up \& go" test as a predictor of falls within 6 months after hip fracture surgery. Phys Ther 2007, 87:24-30.

12. Hale WA, Delaney MJ, McGaghie WC: Characteristics and predictors of falls in elderly patients. J Fam Pract 1992, 34:577-581.

13. Tinetti ME: Performance-oriented assessment of mobility problems in elderly patients. J Am Geriatr Soc 1986, 34:119-126.

14. Narayanan MR, Scalzi ME, Redmond SJ, Lord SR, Celler BG, Lovell NH: A wearable triaxial accelerometry system for longitudinal assessment of falls risk. Conf Proc IEEE Eng Med Biol Soc 2008, 2008:2840-2843.

15. Narayanan MR, Lord SR, Budge MM, Celler BG, Lovell NH: Falls management: detection and prevention, using a waist-mounted triaxial accelerometer. Conf Proc IEEE Eng Med Biol Soc 2007, 2007:4037-4040. 
16. Marschollek M, Nemitz G, Gietzelt M, Wolf KH, Meyer Zu, Schwabedissen H, Haux R: Predicting in-patient falls in a geriatric clinic: a clinical study combining assessment data and simple sensory gait measurements. Z Gerontol Geriatr 2009, 42:317-321.

17. Gietzelt M, Nemitz G, Wolf KH, Meyer Zu Schwabedissen H, Haux R, Marschollek M: A clinical study to assess fall risk using a single waist accelerometer. Inform Health Soc Care 2009, 34:181-188.

18. Marschollek M, Rehwald A, Wolf KH, Gietzelt M, Nemitz G, Meyer Zu Schwabedissen H, Haux R: Sensor-based Fall Risk Assessment - an Expert 'to go'. Methods Inf Med 2011, 50.

19. Marschollek M, Wolf KH, Gietzelt M, Nemitz G, Meyer Zu Schwabedissen $H_{4}$ Haux R: Assessing elderly persons' fall risk using spectral analysis on accelerometric data-a clinical evaluation study. Conf Proc IEEE Eng Med Biol Soc 2008, 2008:3682-3685.

20. Mahoney Fl, Barthel DW: Functional Evaluation: The Barthel Index. Md State Med J 1965, 14:61-65.

21. Marschollek M, Rehwald A, Gietzelt M, Song B, Wolf KH, Haux R: Daily activities and fall risk - A follow-up study to identify relevant activities for sensor-based fall risk assessment. Stud Health Technol Inform 2010, 160:68-72.

22. Lamb SE, Jorstad-Stein EC, Hauer K, Becker C: Development of a common outcome data set for fall injury prevention trials: the Prevention of Falls Network Europe consensus. J Am Geriatr Soc 2005, 53:1618-1622.

23. Witten $\mid H$, Frank E: Data Mining: Practical Machine Learning Tools and Techniques, Second Edition (Morgan Kaufmann Series in Data Management Systems) Morgan Kaufmann Publishers Inc.; 2005.

24. Ikeda $M$, Itoh $S$, Ishigaki T, Yamauchi $K$ : Application of resampling techniques to the statistical analysis of the Brier score. Methods Inf Med 2001, 40:259-264.

25. Kim EA, Mordiffi SZ, Bee WH, Devi K, Evans D: Evaluation of three fall-risk assessment tools in an acute care setting. J Adv Nurs 2007, 60:427-435.

26. Nordin E, Lindelof N, Rosendahl E, Jensen J, Lundin-Olsson L: Prognostic validity of the Timed Up-and-Go test, a modified Get-Up-and-Go test, staff's global judgement and fall history in evaluating fall risk in residential care facilities. Age Ageing 2008, 37:442-448.

27. Thrane $\mathrm{G}$, Joakimsen RM, Thornquist E: The association between timed up and go test and history of falls: the Tromso study. BMC Geriatr 2007, 7:1.

28. Lord SR, Menz HB, Tiedemann A: A physiological profile approach to falls risk assessment and prevention. Phys Ther 2003, 83:237-252.

29. Marschollek M: Recent progress in sensor-enhanced health information systems - slowly but sustainably. Inform Health Soc Care 2009, 34:225-230.

30. Van Hoecke S, Volckaert B, Dhoedt B, De Turck F: Large-scale Performance Evaluation of e-Homecare Architectures Using the WS-NS Simulator. Methods Inf Med 2010, 49.

31. Mancini F, Sousa FS, Hummel AD, Falcao AE, Yi LC, Ortolani CF, Sigulem D, Pisa IT: Classification of Postural Profiles among Mouth-breathing Children by Learning Vector Quantization. Methods Inf Med 2010, 49.

32. Hoerbst A, Ammenwerth E: Electronic health records. A systematic review on quality requirements. Methods Inf Med 2010, 49:320-336.

33. Pecchia L, Bath PA, Pendleton N, Bracale M: Analytic Hierarchy Process (AHP) for Examining Healthcare Professionals' Assessments of Risk Factors. The Relative Importance of Risk Factors for Falls in Communitydwelling Older People. Methods Inf Med 2010, 50.

34. Mathie MJ, Coster AC, Lovell NH, Celler BG, Lord SR, Tiedemann A: A pilot study of long-term monitoring of human movements in the home using accelerometry. J Telemed Telecare 2004, 10:144-151.

35. Marschollek M, Ludwig W, Schapiewski I, Schriever E, Schubert R, Dybowski H, Meyer Zu Schwabedissen H, Howe J, Haux R: Multimodal Home Monitoring of Elderly People-First Results from the LASS Study. In Proceedings - 21st International Conference on Advanced Information Networking an Applications. Volume 2. Niagara Falls, CA: IEEE Computer Society; 2007:815-819.

36. Marschollek M, Wolf KH, Plischke M, Ludwig W, Haux R, Mihailidis A, Howe J: People's perceptions and expectations of assistive healthenabling technologies: an empirical study in Germany. Assist Technol 2009, 21:86-93.

37. Fensli R, Pedersen PE, Gundersen T, Hejlesen O: Sensor acceptance model - measuring patient acceptance of wearable sensors. Methods Inf Med 2008, 47:89-95.

\section{Pre-publication history}

The pre-publication history for this paper can be accessed here: http://www.biomedcentral.com/1472-6947/11/48/prepub

doi:10.1186/1472-6947-11-48

Cite this article as: Marschollek et al:: Sensors vs. experts - A performance comparison of sensor-based fall risk assessment vs. conventional assessment in a sample of geriatric patients. BMC Medical Informatics and Decision Making 2011 11:48.

\section{Submit your next manuscript to BioMed Central and take full advantage of:}

- Convenient online submission

- Thorough peer review

- No space constraints or color figure charges

- Immediate publication on acceptance

- Inclusion in PubMed, CAS, Scopus and Google Scholar

- Research which is freely available for redistribution

Submit your manuscript at www.biomedcentral.com/submit
Ciomed Central 